\title{
BMJ Open Association between $C$ reactive protein level and depressive symptoms in an elderly Korean population: Korean Social Life, Health and Aging Project
}

\author{
Bo Mi Song, ${ }^{1,2}$ Ju-Mi Lee, ${ }^{2,3}$ Wungrak Choi, ${ }^{4}$ Yoosik Youm, ${ }^{5}$ Sang Hui Chu, ${ }^{6}$ \\ Yeong-Ran Park, ${ }^{7}$ Hyeon Chang Kim ${ }^{2,3}$
}

To cite: Song BM, Lee J-M, Choi W, et al. Association between $\mathrm{C}$ reactive protein level and depressive symptoms in an elderly Korean population: Korean Social Life, Health and Aging Project. BMJ Open 2015;5: e006429. doi:10.1136/ bmjopen-2014-006429

- Prepublication history and additional material is available. To view please visit the journal (http://dx.doi.org/ 10.1136/bmjopen-2014006429).

Received 20 August 2014 Revised 23 January 2015 Accepted 3 February 2015

CrossMark

For numbered affiliations see end of article.

Correspondence to Dr Hyeon Chang Kim; hckim@yuhs.ac

\section{ABSTRACT}

Objective: The relationship between inflammatory markers and depressive symptoms has been reported inconsistently. Moreover, there were only a few studies conducted in an Asian population. The purpose of this study was to examine the association between $C$ reactive protein (CRP) and depressive symptoms in an elderly Korean population.

Design, setting and participants: This study used data from the Korean Social Life, Health and Aging Project Health Examination Cohort, which started in 2011. Among participants aged 60 or over recruited from a rural community, 569 (224 men and 345 women) without a history of stroke, angina pectoris, myocardial infarction or CRP $\geq 20 \mathrm{mg} / \mathrm{L}$ were employed for cross-sectional analyses. As a marker of systemic inflammation, CRP was measured. Depressive symptoms were measured with the Center for Epidemiologic Studies Depression (CES-D) scale. Multiple linear regression analysis was used to investigate the relationship between CRP and depressive symptoms.

Results: In men, CRP levels had significant associations with depressive symptoms before $(\beta=0.420, p=0.010)$ and after $(\beta=0.336, p=0.025)$ adjusting for age, body mass index, systolic blood pressure, number of comorbidities, smoking status, alcohol intake, marital status, education and sleep duration. However, in women, the association between CRP and depressive symptoms was not significant before $(p=0.250)$ and after $(p=0.256)$ adjustment. Conclusions: Our findings suggest that elevated CRP levels are independently associated with the presence of depressive symptoms in elderly Korean men.

\section{INTRODUCTION}

The elderly population is growing worldwide and has been accompanied by a concurrent increase in physical or psychological disabilities. ${ }^{1}$ Depression is among the highly prevalent disorders in elderly people, and leads to increased risk of mortality. ${ }^{2}$ The underlying

\section{Strengths and limitations of this study}

- We controlled sociodemographic, lifestyle, physical and psychological factors to evaluate the independent association between serum CRP levels and depressive symptoms.

- This study is the first to demonstrate that serum CRP levels are associated with depressive symptoms in elderly Koreans.

- Limitations include a single measurement of serum CRP and use of self-report questionnaires for depressive symptoms.

mechanisms for depressive symptoms in old age remain unclear, but the inflammatory host response is repeatedly inferred in the pathogenesis of neuropsychiatric conditions. ${ }^{3}{ }^{4}$ In several population studies including older participants, the relationship between inflammatory markers and depressive symptoms has been described. ${ }^{2}{ }^{5-13}$ However, those studies have generated conflicting results. Some studies observed positive associations between inflammatory markers and depressive symptoms, ${ }^{2}{ }^{5-9} 11$ but others did not. ${ }^{12}{ }^{13}$ This inconsistency might be due to differences in study population, measurements of depression and control for important confounders. Moreover, most of the reported data are based on studies conducted in western countries, with only a few exceptions targeting the Asian population. ${ }^{12} 14$

Thus, we investigated the independent association between serum $\mathrm{C}$ reactive protein (CRP), a marker of systemic inflammation, and depressive symptoms in an elderly Korean population.

\section{METHODS}

Study population

The present study used data from the Korean Social Life, Health and Aging Project 
(KSHAP), which is a population-based longitudinal study of health determinants among elderly Koreans. The target populations of the KSHAP are people aged 60 years or older and their spouses living within one township (myeon) of Ganghwa-gun, Incheon, Korea. This township is a typical rural Korean village where farming is the main industry. A complete enumeration survey was conducted in the first wave of the KSHAP on $94.7 \%(n=814)$ of the target population $(n=860)$ between December 2011 and July 2012. The KSHAP-Health Examination (KSHAP-HE) cohort consists of 698 people who completed additional health examinations at a public health centre or at their home. The sampling and data collection procedures have been described in detail elsewhere. ${ }^{15}$ In this study, 129 participants were excluded for missing key variables $(\mathrm{n}=85)$; history of stroke, angina pectoris or myocardial infarction $(n=32)$; or CRP levels above $20 \mathrm{mg} / \mathrm{L}(\mathrm{n}=12)$. Ultimately, a total of 569 participants (224 men and 345 women) were included for crosssectional analyses. All participants provided written informed consent forms.

\section{Questionnaire data}

All participants were interviewed by trained personnel using standardised questionnaires according to the predeveloped protocol. In addition, the designated field director double-checked whether answers were missing or inappropriate and continuously monitored the entire interview process. Sociodemographic variables included age, education (uneducated, elementary school, middle school or high school+) and marital status (with or without spouse). Health behaviours included smoking status, alcohol intake and sleep duration. Smoking status was classified into two groups: current smokers or current non-smokers (past smokers or never smokers). Alcohol intake was categorised as follows: regular alcohol drinkers or others (participants who drink under once a week or never alcohol drinkers). Sleep duration was collected in hours per day $(\mathrm{h} /$ day) as the average during the past year. The presence of comorbidities were assessed by self-report and included diseases as follows: hypertension, diabetes mellitus, metabolic syndrome, dyslipidaemia, osteoporosis, cancer, stroke, myocardial infarction, angina pectoris, arthritis, pulmonary tuberculosis, asthma, cataract, glaucoma, hepatitis B, depression, urinary incontinence and benign prostatic hyperplasia. Depressive symptoms were measured using the Korean version of the Center for Epidemiologic Studies Depression (CES-D) scale, a 20-item, self-report scale designed to measure depressive symptoms experienced during the previous week. ${ }^{16}$ The scale, ranging from 0 to 60 , has been shown to be a valid and reliable instrument in older populations. ${ }^{17}$ The underlying structure of the CES-D consists of four dimensions of depression: seven items measure depressed affect, four items measure positive affect, two items measure interpersonal difficulties and seven items measure somatic symptoms.

\section{Physical examination}

All participants wore lightweight hospital gowns for convenient and reliable examinations. Standing height was measured to the nearest $0.1 \mathrm{~cm}$ using a stadiometer and body weight was measured to the nearest $0.1 \mathrm{~kg}$ on a digital scale. Body mass index (BMI) was calculated as body weight in kilograms divided by standing height in metres squared $\left(\mathrm{kg} / \mathrm{m}^{2}\right)$. Participants were seated for at least 5 min before blood pressure measurement, and two measurements were taken within at least 5 min using an automatic sphygmomanometer (Dinamap 1846 SX/P; GE Healthcare, Waukesha, Wisconsin, USA). If the two measurements differed by $\geq 10 \mathrm{~mm} \mathrm{Hg}$ for systolic blood pressure (SBP) or diastolic blood pressure (DBP), a third measurement was taken after $5 \mathrm{~min}$, and the last two measurements were averaged for analysis.

\section{Laboratory test}

Blood samples were collected from the antecubital vein of participants after at least $8 \mathrm{~h}$ of fasting. Collected blood samples were analysed at a central research laboratory for serum CRP, total cholesterol, high-density lipoprotein cholesterol, triglycerides, glucose and so on. CRP levels, a marker of acute systemic inflammation, were determined in accordance with turbidimetric immunoassay with an ADVIA1800 Auto Analyzer (Siemens medical Sol., USA). The interassay coefficient of variation for CRP was from $0.38 \%$ to $1.53 \%$ and results were expressed as $\mathrm{mg} / \mathrm{L}$.

\section{Statistical analysis}

We evaluated differences in clinical and demographic characteristics between men and women. CRP level, CES-D scale and several confounding factors were significantly different between men and women. Therefore, we performed all analyses separately by sex. Continuous variables were described as mean and SD (for normally distributed variables) or as median and IQR (for skewed variables), and tested by independent $t$ test and Wilcoxon rank sum test, respectively. Categorical variables were described as numbers with percentage and tested by a $\chi^{2}$ test. General characteristics and concentrations of selected biomarkers were also analysed according to three categories of depression score. One-way analysis of variance was used for continuous variables and $\chi^{2}$ test was used for categorical variables. For this analysis, the trend test was used. For the continuous variables, $p$ values were calculated by using a contrast to test for a linear trend. For the categorical variables, the Cochran-Armitage test and the Mantel-Haenszel test were used. The relationships between depression score and other variables were evaluated using Pearson's correlation coefficients. Multiple linear regression analyses were used to assess the independent association between serum CRP and depression score in the unadjusted model and two adjusted models: (1) adjusting for age, BMI, SBP, number of comorbidities, smoking status and alcohol intake; (2) additional adjustment for marital status, sleep duration 
and education. All statistical analyses were performed using SAS V.9.2 (SAS Institute, Cary, North Carolina, USA), and statistical significance was defined as two-sided $\mathrm{p}$ value less than 0.05 .

\section{RESULTS}

Clinical and demographic characteristics of the study participants are presented in table 1 . This study consisted of 224 men with a mean age of 72.8 years and 345 women with a mean age of 71.6 years. The median CRP level was significantly higher in men than in women (1.0 vs $0.8 \mathrm{mg} / \mathrm{L}, \mathrm{p}<0.001)$, but the median CES-D scale was significantly lower in men than in women (6 vs 8 , $\mathrm{p}=0.007$ ).

Table 2 shows the characteristics of the study participants according to the CES-D tertile. Both men and women with higher CES-D categories tended to be older and have more number of comorbidities. In men, higher CES-D categories were associated with higher CRP level $(p=0.038)$, lower education level and no spouse (divorced, widowed and unmarried). However, in women, the CED-D tertile was not associated with the CRP level $(\mathrm{p}=0.465)$.

Table 3 shows correlations between depression score and other variables. In the unadjusted model, depression score was positively associated with age, SBP, number of comorbidities and CRP in men. In women, depression score was positively associated with age and number of comorbidities; however, the association with CRP was not significant. Figure 1 presents the nature of relationship between depression score and logtransformed CRP, separately for men and women.

Table 4 presents the association between depression score and serum CRP in serial multiple linear regression models. In men, higher CRP levels were significantly associated with increased depression score $(\beta=0.365$, $\mathrm{p}=0.017$ ) when adjusted for age, BMI, SBP, number of comorbidities, smoking status and alcohol intake (model 1). The association still remained significant $(\beta=0.336, \quad p=0.025)$ when additionally adjusted for marital status, sleep duration and education (model 2). However, in women, the associations between CRP level and depression score were not significant in the unadjusted model and two adjusted models.

\section{DISCUSSION}

This study investigated the independent association between serum CRP levels and depressive symptoms in an elderly Korean population. Our findings suggest that elevated levels of CRP are independently associated with depressive symptoms in elderly Korean men.

Our results are consistent with previous findings linking elevated CRP levels to depression. ${ }^{5} 911{ }^{18}$ In the

Table 1 Characteristics of study participants

\begin{tabular}{|c|c|c|c|c|}
\hline Variables & Total $(n=569)$ & Men $(n=224)$ & Women $(n=345)$ & p Value \\
\hline Age, year & $72.1 \pm 7.6$ & $72.8 \pm 6.7$ & $71.6 \pm 8.2$ & 0.070 \\
\hline Body mass index, $\mathrm{kg} / \mathrm{m}^{2}$ & $24.0 \pm 3.4$ & $23.7 \pm 3.5$ & $24.2 \pm 3.4$ & 0.073 \\
\hline Systolic BP, $\mathrm{mm} \mathrm{Hg}$ & $134.2 \pm 20.0$ & $133.0 \pm 19.7$ & $135.1 \pm 20.1$ & 0.225 \\
\hline Diastolic BP, mm Hg & $72.1 \pm 9.9$ & $73.2 \pm 10.2$ & $71.5 \pm 9.7$ & 0.047 \\
\hline Total cholesterol, mg/dL & $184.0 \pm 35.1$ & $173.1 \pm 33.5$ & $191.1 \pm 34.4$ & $<0.001$ \\
\hline HDL cholesterol, mg/dL & $51.4 \pm 12.8$ & $51.1 \pm 13.6$ & $51.6 \pm 12.2$ & 0.668 \\
\hline LDL cholesterol, mg/dL & $102.1 \pm 30.8$ & $92.6 \pm 28.6$ & $108.1 \pm 30.6$ & $<0.001$ \\
\hline Triglycerides, mg/dL & 138 [102-189] & $128[93-176]$ & 142 [105-193] & $0.011^{*}$ \\
\hline $\mathrm{CRP}, \mathrm{mg} / \mathrm{L}$ & $0.8[0.5-1.6]$ & $1.0[0.6-2.1]$ & $0.8[0.4-1.4]$ & $<0.001^{*}$ \\
\hline Hypertension & $369(64.9)$ & $139(62.1)$ & $230(66.7)$ & $0.300 \dagger$ \\
\hline Diabetes mellitus & $122(21.4)$ & $53(23.7)$ & $69(20.0)$ & $0.350 \dagger$ \\
\hline Dyslipidaemia & $243(42.7)$ & $86(38.4)$ & $157(45.5)$ & $0.112 \dagger$ \\
\hline Number of comorbidities & $1.9 \pm 1.5$ & $1.4 \pm 1.3$ & $2.2 \pm 1.5$ & $<0.001$ \\
\hline Current smoker & $70(12.3)$ & $64(28.6)$ & $6(1.7)$ & $<0.001 \dagger$ \\
\hline Regular alcohol drinker & $117(20.6)$ & $96(42.9)$ & $21(6.1)$ & $<0.001 \dagger$ \\
\hline Sleep duration, $\mathrm{h}$ & $8.5 \pm 1.4$ & $8.4 \pm 1.4$ & $8.5 \pm 1.4$ & 0.201 \\
\hline \multicolumn{5}{|l|}{ Education } \\
\hline Uneducated & $167(29.6)$ & $25(11.2)$ & $142(41.6)$ & \multirow[t]{4}{*}{$<0.001 \dagger$} \\
\hline Elementary school & $240(42.6)$ & $97(43.5)$ & $143(41.9)$ & \\
\hline Middle school & $83(14.7)$ & $52(23.3)$ & $31(9.1)$ & \\
\hline High school or higher & $74(13.1)$ & $49(22.0)$ & $25(7.3)$ & \\
\hline No spouse (divorced, widowed and unmarried) & $138(24.3)$ & $16(7.1)$ & $122(35.4)$ & $<0.001 \dagger$ \\
\hline CES-D & 7 [4-14] & 6 [3-13] & 8 [4-15] & $0.007^{\star}$ \\
\hline CES-D $\geq 16$ & $124(21.8)$ & $41(18.3)$ & $83(24.1)$ & $0.128 \dagger$ \\
\hline \multicolumn{5}{|c|}{$\begin{array}{l}\text { Data are expressed as means } \pm \text { SD, medians [IQR] or numbers }(\%) \text {. } \\
\text { LDL cholesterol levels were calculated for } 558 \text { participants ( } 217 \text { men and } 341 \text { women). } \\
\text { Education levels were measured for } 564 \text { participants ( } 223 \text { men and } 341 \text { women). } \\
\text { p Value was derived from an independent } t \text { test, Wilcoxon rank sum test }{ }^{*} \text { or } \chi^{2} \text { testt. } \\
\text { Abbreviations: BP, blood pressure; CES-D, Center for Epidemiologic Studies Depression; CRP, C reactive protein; HDL, high-density } \\
\text { lipoprotein; LDL, low-density lipoprotein. }\end{array}$} \\
\hline
\end{tabular}


Table 2 Characteristics of study participants by CES-D tertile

\begin{tabular}{|c|c|c|c|c|c|c|c|c|}
\hline \multirow[t]{2}{*}{ Variables } & \multicolumn{4}{|l|}{ Men $(n=224)$} & \multicolumn{4}{|c|}{ Women $(n=345)$} \\
\hline & $0-3(n=65)$ & $4-10(n=85)$ & $11+(n=74)$ & p Trend & $0-5(n=123)$ & $6-12(n=107)$ & $13+(n=115)$ & p Trend \\
\hline Age, year & $69.7 \pm 5.8$ & $73.0 \pm 6.2$ & $75.2 \pm 7.1$ & $<0.001$ & $70.8 \pm 8.3$ & $71.1 \pm 7.4$ & $73.1 \pm 8.5$ & 0.031 \\
\hline Body mass index, $\mathrm{kg} / \mathrm{m}^{2}$ & $24.0 \pm 3.9$ & $24.0 \pm 3.1$ & $23.1 \pm 3.4$ & 0.124 & $24.2 \pm 3.3$ & $24.4 \pm 3.3$ & $24.0 \pm 3.5$ & 0.685 \\
\hline Systolic BP, mm Hg & $133.1 \pm 20.1$ & $130.1 \pm 18.7$ & $136.2 \pm 20.4$ & 0.358 & $137.9 \pm 22.1$ & $132.6 \pm 17.6$ & $134.4 \pm 19.9$ & 0.178 \\
\hline Diastolic BP, mm Hg & $75.2 \pm 11.1$ & $72.0 \pm 9.4$ & $72.7 \pm 10.2$ & 0.156 & $72.6 \pm 9.9$ & $71.8 \pm 9.1$ & $70.0 \pm 9.9$ & 0.038 \\
\hline Total cholesterol, mg/dL & $179.7 \pm 38.3$ & $176.1 \pm 30.7$ & $163.9 \pm 30.1$ & 0.005 & $191.1 \pm 35.6$ & $189.7 \pm 33.3$ & $192.3 \pm 34.3$ & 0.798 \\
\hline HDL cholesterol, mg/dL & $50.4 \pm 13.0$ & $51.2 \pm 11.7$ & $51.6 \pm 16.1$ & 0.603 & $51.3 \pm 12.0$ & $50.8 \pm 11.7$ & $52.5 \pm 12.9$ & 0.467 \\
\hline LDL cholesterol, mg/dL & $95.7 \pm 29.0$ & $95.9 \pm 29.4$ & $86.3 \pm 26.5$ & 0.059 & $107.2 \pm 30.9$ & $107.4 \pm 30.4$ & $109.7 \pm 30.8$ & 0.539 \\
\hline Triglycerides, mg/dL & $131[97-187]$ & 135 [94-193] & $120[89-150]$ & 0.009 & $146[106-201]$ & $141[107-188]$ & 142 [99-193] & 0.418 \\
\hline CRP, mg/L & $0.8[0.5-1.5]$ & $1.3[0.6-2.2]$ & $1.1[0.5-3.0]$ & 0.038 & $0.8[0.4-1.3]$ & $0.7[0.5-1.3]$ & $0.8[0.4-1.6]$ & 0.465 \\
\hline Hypertension & $34(52.3)$ & $52(61.2)$ & $53(71.6)$ & $0.019^{*}$ & $85(69.1)$ & $71(66.4)$ & $74(64.4)$ & $0.436^{*}$ \\
\hline Diabetes mellitus & $14(21.5)$ & $17(20.0)$ & $22(29.7)$ & $0.241^{*}$ & $19(15.5)$ & $22(20.6)$ & $28(24.4)$ & $0.086^{*}$ \\
\hline Dyslipidaemia & $26(40.0)$ & $40(47.1)$ & $20(27.0)$ & $0.099^{\star}$ & 57 (46.3) & $50(46.7)$ & $50(43.5)$ & $0.662^{*}$ \\
\hline Number of comorbidities & $1.0 \pm 1.3$ & $1.5 \pm 1.2$ & $1.8 \pm 1.4$ & $<0.001$ & $1.8 \pm 1.4$ & $2.2 \pm 1.5$ & $2.5 \pm 1.6$ & 0.001 \\
\hline Current smoker & $18(27.7)$ & $22(25.9)$ & $24(32.4)$ & $0.146 \dagger$ & $3(2.4)$ & $1(0.9)$ & $2(1.7)$ & $0.669+$ \\
\hline Regular alcohol drinker & $28(43.1)$ & $36(42.4)$ & $32(43.2)$ & $0.572 \dagger$ & $6(4.9)$ & $9(8.4)$ & $6(5.2)$ & $0.892 \dagger$ \\
\hline Sleep duration, $\mathrm{h}$ & $8.2 \pm 1.2$ & $8.5 \pm 1.5$ & $8.5 \pm 1.5$ & 0.218 & $8.5 \pm 1.4$ & $8.4 \pm 1.5$ & $8.7 \pm 1.4$ & 0.391 \\
\hline \multicolumn{9}{|l|}{ Education } \\
\hline Uneducated & $4(6.2)$ & $8(9.4)$ & $13(17.8)$ & $<0.001 \dagger$ & $45(36.6)$ & $48(45.3)$ & $49(43.8)$ & $0.054 †$ \\
\hline Elementary school & $24(36.9)$ & $39(45.9)$ & $34(46.6)$ & & $54(43.9)$ & $40(37.7)$ & $49(43.8)$ & \\
\hline Middle school & 17 (26.2) & $16(18.8)$ & $19(26.0)$ & & $9(7.3)$ & 13 (12.3) & $9(8.0)$ & \\
\hline High school or higher & $20(30.8)$ & $22(25.9)$ & $7(9.6)$ & & $15(12.2)$ & $5(4.7)$ & $5(4.5)$ & \\
\hline No spouse (divorced, widowed and unmarried) & $0(0.0)$ & $4(4.7)$ & $12(16.2)$ & $<0.001^{*}$ & $42(34.2)$ & $34(31.8)$ & $46(40.0)$ & $0.354^{*}$ \\
\hline
\end{tabular}


Table 3 Pearson's correlation coefficients between depression score and other variables

\begin{tabular}{|c|c|c|c|c|}
\hline \multirow[b]{2}{*}{ Variables } & \multicolumn{2}{|l|}{ Men $(n=224)$} & \multicolumn{2}{|l|}{ Women $(n=345)$} \\
\hline & Correlation coefficient & p Value & Correlation coefficient & p Value \\
\hline Age & 0.333 & $<0.001$ & 0.177 & $<0.001$ \\
\hline Body mass index & -0.099 & 0.142 & -0.064 & 0.236 \\
\hline Systolic BP & 0.144 & 0.031 & -0.015 & 0.785 \\
\hline Diastolic BP & 0.020 & 0.771 & -0.061 & 0.261 \\
\hline Sleep duration & 0.045 & 0.506 & 0.050 & 0.359 \\
\hline Number of comorbidities & 0.268 & $<0.001$ & 0.232 & $<0.001$ \\
\hline $\mathrm{CRP}^{*}$ & 0.140 & 0.036 & 0.055 & 0.312 \\
\hline
\end{tabular}

${ }^{*}$ Analysed with log-transformed values.

$\mathrm{BP}$, blood pressure; CRP, $\mathrm{C}$ reactive protein.

Health, Aging and Body Composition Study, high levels of inflammatory markers including CRP, interleukin 6 (IL-6) and tumour necrosis factor $\alpha(\mathrm{TNF}-\alpha)$ were associated with depressive symptoms which are measured with the CES-D scale. ${ }^{5}$ Moreover, in older people with metabolic syndrome, CRP levels were independently associated with depressive symptoms after adjusting for sex, age, education, smoking status, cognitive function, metabolic syndrome, hypertension, diabetes and

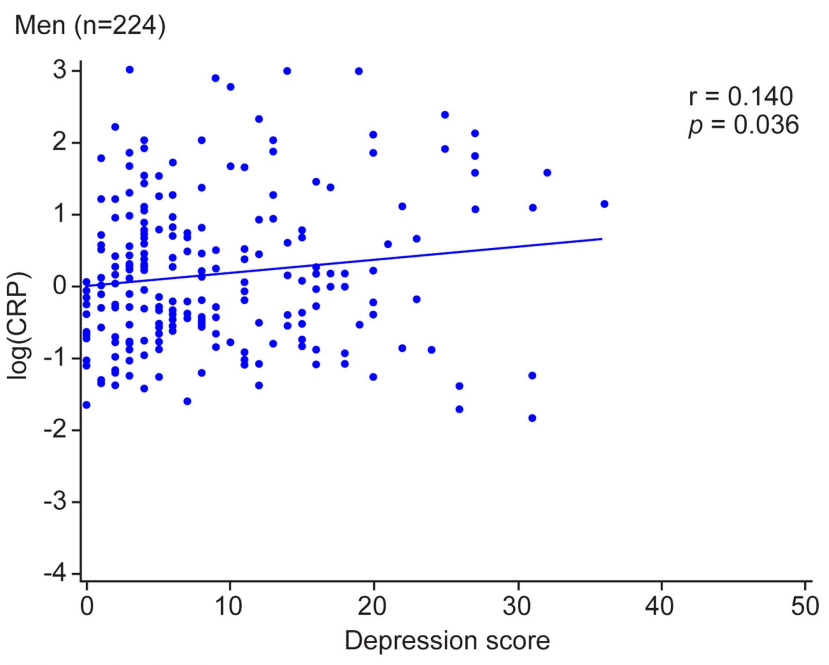

Women $(n=345)$

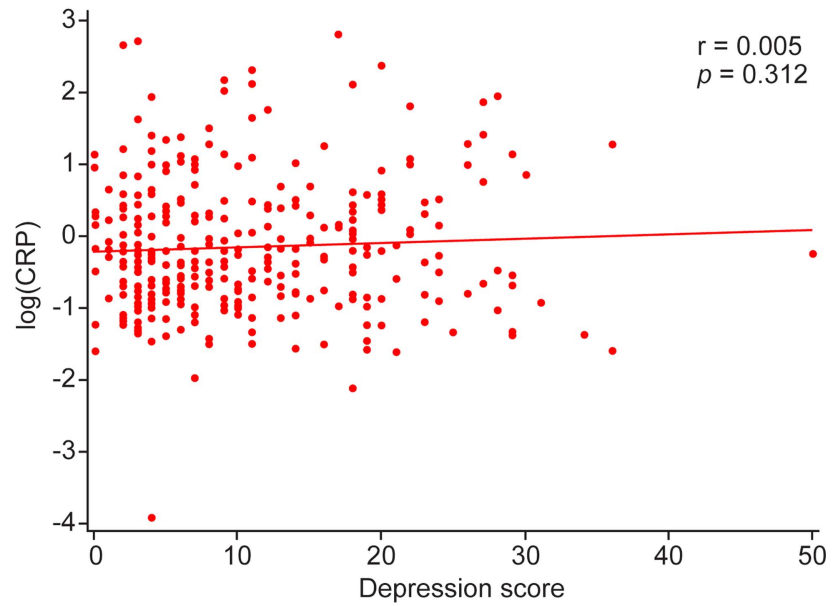

Figure 1 Association score between depression score and $\mathrm{C}$ reactive protein (CRP). medication. ${ }^{11}$ Lastly, a systemic review and meta-analysis of longitudinal studies which are conducted across all age groups found that raised inflammatory markers including CRP and IL-6 have a small but significant association with the subsequent development of depressive symptoms after adjustment for age and a wide range of factors associated with risk for depression. ${ }^{9}$ Contrary to these studies, however, several studies did not find independent associations between CRP levels and depressive symptoms. ${ }^{10} 19{ }^{20}$ In two elderly population studies, elevated CRP levels were associated with higher CES-D score, but not after adjustment for confounding factors. ${ }^{10} 19$ In the prospective Sydney Memory and Aging Study, CRP levels were not associated with depressive symptoms, whereas IL- 8 was associated with depressive symptoms at baseline and at 2-year follow-up. ${ }^{20}$

The relationship between inflammation and depression is potentially bidirectional, and the underlying mechanisms are still poorly understood. First, inflammation may lead to depression. Increased inflammatory markers contribute to decreases in serotonin, an important neurotransmitter in the pathogenesis of depression. ${ }^{21-23}$ Second, depression may lead to inflammation. Psychological stress activates the sympathetic nervous system and releases stress hormones. These hormones in turn initiate acute-phase responses triggering inflammation. ${ }^{24-26}$

In our study, CRP levels were significantly associated with depressive symptoms in men but not in women. This finding is consistent with a few previous reports. ${ }^{27-29} \mathrm{In}$ an obese clinical population, depressive symptoms were significantly associated with CRP in men only, even after adjusting for age, obesity, metabolic variables and medications known to affect inflammation. This finding suggests that there are biological differences between men and women that may modify the relationship between CRP levels and depression. ${ }^{28}$ In the third National Health and Nutrition Examination Survey (NHANES III), depression and elevated CRP levels were more common in women than in men, but the association between CRP levels and depression was significant only in men. ${ }^{29}$ They hypothesised that CRP levels might vary by the hormonal environment. In the Health 2000 Survey, depressive symptoms were independently associated with systemic inflammation processes in men, but not in women. ${ }^{27}$ They suggest that 


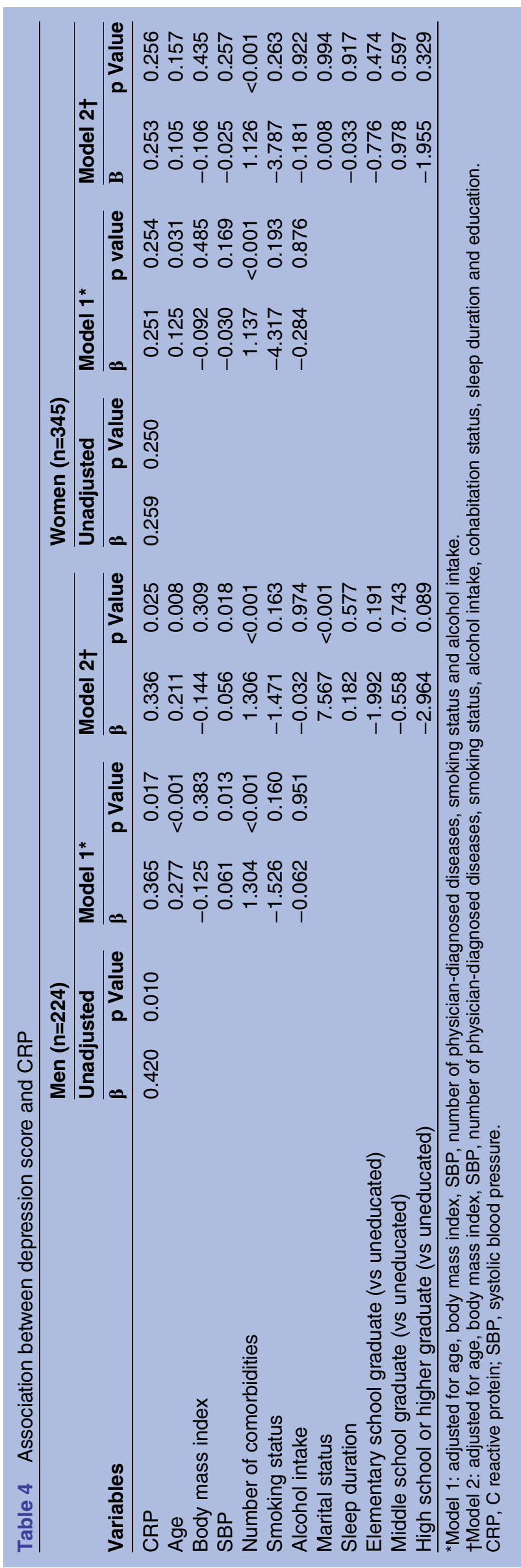

depression may have a different psychological and physiological meaning and background for men and women. Our study participants were the elderly population aged 60 years or older. Therefore, our findings that there was a significant association between CRP levels and depressive symptoms in men only are probably because the main factors influencing on depression differ rather than hormonal environments or biological features differ between men and women.

Our study has a few distinctions compared with previous studies. The study participants were recruited from a community of single ethnic background. Furthermore, we carefully controlled various covariates known to be related to inflammation and depression in the analyses. These enabled us to evaluate the independent association between the inflammatory marker and depressive symptoms. To the best of our knowledge, this study is the first to demonstrate that serum CRP levels are associated with depressive symptoms in elderly Koreans. We also observed the gender difference in the association between CRP levels and depressive symptoms among general elderly Koreans.

The study has some limitations. First, since this is a crosssectional study in which all information was collected at the same point in time, no definitive conclusion about the direction of the associations between CRP levels and depressive symptoms could be reached. Second, we relied on a single blood sample per individual. However, circulating CRP levels obtained from one time point have been shown to represent systemic low-grade inflammation. ${ }^{30}$ Third, inflammatory markers other than CRP were not measured in this study. Further studies including other inflammatory markers needed to confirm our results. Fourth, we investigated depressive symptoms using the CES-D scale, which has been widely used to assess depression and its risk factors in population-based studies. ${ }^{17} 16$ However, the validity of CES-D is inferior to the psychiatric diagnostic interview, the gold standard. ${ }^{31}$ Fifth, we did not use a validated measure of comorbidity such as the Charlson Comorbidity index because comorbidity components of the Charlson Comorbidity index were not matched with the comorbidities asked for in this study. However, many epidemiological studies have used a number of selected chronic disorders to measure general health conditions of elderly people. ${ }^{120}{ }^{32}$ Sixth, we could not control the effects of medications such as hormonereplacement therapy, anti-inflammatory medications and antidepressants. Alternatively, we additionally analysed after excluding 11 people with a history of depression. A similar result was observed (online supplementary table S1). Lastly, the study participants were recruited from a single rural community and the sample size was relatively small. Consequently, our findings may not be generalisable to the other older population.

In conclusion, our findings suggest that high serum CRP levels are independently associated with depressive symptoms in community-dwelling elderly Korean men, even after adjusting for several physical and psychological 
factors. Chronic inflammation may be another possible risk factor for depressive symptoms in elderly Koreans. Further studies are needed to establish a causal relationship between inflammation and depression and to investigate the underlying mechanisms by sex and specific population.

\author{
Author affiliations \\ ${ }^{1}$ Department of Public Health, Yonsei University Graduate School, Seoul, \\ Korea \\ ${ }^{2}$ Cardiovascular and Metabolic Disease Etiology Research Center, Yonsei \\ University College of Medicine, Seoul, Korea \\ ${ }^{3}$ Department of Preventive Medicine, Yonsei University College of Medicine, \\ Seoul, Korea \\ ${ }^{4}$ Department of Ophthalmology, Yonsei University College of Medicine, Seoul, \\ Korea \\ ${ }^{5}$ Department of Sociology, Yonsei University College of Social Sciences, \\ Seoul, Korea \\ ${ }^{6}$ Department of Clinical Nursing Science, Yonsei University College of \\ Nursing, Seoul, Korea \\ ${ }^{7}$ Division of Silver Industry, Kangnam University, Yongin, Korea
}

Acknowledgements The authors thank all the staff participating in the project.

Contributors YY, SHC, Y-RP and HCK designed the research; J-ML and WC supervised the study and acquired data; BMS analysed the data and wrote the paper; J-ML and HCK provided a critical revision of the manuscript for important intellectual content; and HCK had a primary responsibility for the final content. All authors read and approved the final manuscript.

Funding This study was supported by grants from the National Research Foundation of Korea Grant (NRF-2011-330-B00137) and the Korean Health Technology R\&D Project, Ministry of Health \& Welfare (HI13C0715), Republic of Korea.

Competing interests None.

Ethics approval This study was approved by the Institutional Review Board of Yonsei University (YUIRB-2011-012-01).

Provenance and peer review Not commissioned; externally peer reviewed.

Data sharing statement Collaboration is encouraged and access to the KSHAP baseline data is available via the website of the Korean Social Science Data Archive (http://www.kossda.or.kr).

Open Access This is an Open Access article distributed in accordance with the Creative Commons Attribution Non Commercial (CC BY-NC 4.0) license, which permits others to distribute, remix, adapt, build upon this work noncommercially, and license their derivative works on different terms, provided the original work is properly cited and the use is non-commercial. See: http:// creativecommons.org/licenses/by-nc/4.0/

\section{REFERENCES}

1. Adriaensen W, Mathei C, van Pottelbergh G, et al. Significance of serum immune markers in identification of global functional impairment in the oldest old: cross-sectional results from the BELFRAIL study. Age (Dordr) 2014;36:457-67.

2. van den Biggelaar $\mathrm{AH}$, Gussekloo J, de Craen AJ, et al. Inflammation and interleukin-1 signaling network contribute to depressive symptoms but not cognitive decline in old age. Exp Gerontol 2007;42:693-701.

3. Anisman H, Merali Z. Cytokines, stress and depressive illness: brain-immune interactions. Ann Med 2003;35:2-11.

4. Raison $\mathrm{CL}$, Capuron L, Miller AH. Cytokines sing the blues: inflammation and the pathogenesis of depression. Trends Immunol 2006:27:24-31.

5. Penninx BW, Kritchevsky SB, Yaffe K, et al. Inflammatory markers and depressed mood in older persons: results from the Health, Aging and Body Composition study. Biol Psychiatry 2003;54:566-72.

6. Dimopoulos N, Piperi C, Psarra V, et al. Increased plasma levels of 8-iso-PGF2alpha and IL-6 in an elderly population with depression. Psychiatry Res 2008;161:59-66.
7. Howren MB, Lamkin DM, Suls J. Associations of depression with C-reactive protein, IL-1, and IL-6: a meta-analysis. Psychosom Med 2009;71:171-86.

8. Einvik G, Flyvbjerg A, Hrubos-Strom $\mathrm{H}$, et al. Novel cardiovascular risk markers in depression: no association between depressive symptoms and osteoprotegerin or adiponectin in persons at high risk for sleep apnea. J Affect Disord 2013:145:400-4.

9. Valkanova V, Ebmeier KP, Allan CL. CRP, IL-6 and depression: a systematic review and meta-analysis of longitudinal studies. $J$ Affect Disord 2013;150:736-44.

10. Bremmer MA, Beekman AT, Deeg DJ, et al. Inflammatory markers in late-life depression: results from a population-based study. J Affect Disord 2008:106:249-55.

11. Viscogliosi G, Andreozzi P, Chiriac IM, et al. Depressive symptoms in older people with metabolic syndrome: is there a relationship with inflammation? Int J Geriatr Psychiatry 2013;28:242-7.

12. Pan $\mathrm{A}, \mathrm{Ye} \mathrm{X}$, Franco $\mathrm{OH}$, et al. The association of depressive symptoms with inflammatory factors and adipokines in middle-aged and older Chinese. PLOS ONE 2008;3:e1392.

13. Kop WJ, Gottdiener JS, Tangen CM, et al. Inflammation and coagulation factors in persons $>65$ years of age with symptoms of depression but without evidence of myocardial ischemia. Am J Cardiol 2002;89:419-24.

14. Su S, Miller AH, Snieder $\mathrm{H}$, et al. Common genetic contributions to depressive symptoms and inflammatory markers in middle-aged men: the Twins Heart Study. Psychosom Med 2009;71:152-8.

15. Lee JM, Lee WJ, Kim HC, et al. The Korean social life, health and aging project-health examination cohort. Epidemiol Health 2014;36: e2014003.

16. Radloff LS. The CES-D scale: a self-report depression scale for research in the general population. Appl Psychol Meas 1977;1:385-401.

17. Beekman AT, Deeg DJ, Van Limbeek J, et al. Criterion validity of the Center for Epidemiologic Studies Depression scale (CES-D): results from a community-based sample of older subjects in the Netherlands. Psychol Med 1997;27:231-5.

18. Wium-Andersen MK, Orsted DD, Nielsen SF, et al. Elevated C-reactive protein levels, psychological distress, and depression in 73, 131 individuals. JAMA Psychiatry 2013;70:176-84.

19. Tiemeier $\mathrm{H}$, Hofman A, van Tuijl HR, et al. Inflammatory proteins and depression in the elderly. Epidemiology 2003;14:103-7.

20. Baune BT, Smith E, Reppermund S, et al. Inflammatory biomarkers predict depressive, but not anxiety symptoms during aging: the prospective Sydney Memory and Aging Study Psychoneuroendocrinology 2012;37:1521-30.

21. Miller $\mathrm{AH}$, Maletic $\mathrm{V}$, Raison $\mathrm{CL}$. Inflammation and its discontents: the role of cytokines in the pathophysiology of major depression. Biol Psychiatry 2009;65:732-41.

22. Haroon E, Raison CL, Miller AH. Psychoneuroimmunology meets neuropsychopharmacology: translational implications of the impact of inflammation on behavior. Neuropsychopharmacology 2012;37:137-62.

23. Capuron L, Miller AH. Immune system to brain signaling: neuropsychopharmacological implications. Pharmacol Ther 2011;130:226-38.

24. Kyrou I, Tsigos C. Stress hormones: physiological stress and regulation of metabolism. Curr Opin Pharmacol 2009;9:787-93.

25. Miller GE, Freedland KE, Carney RM, et al. Pathways linking depression, adiposity, and inflammatory markers in healthy young adults. Brain Behav Immun 2003;17:276-85.

26. Gabay C, Kushner I. Acute-phase proteins and other systemic responses to inflammation. N Engl J Med 1999;340:448-54.

27. Elovainio M, Aalto AM, Kivimaki M, et al. Depression and C-reactive protein: population-based Health 2000 Study. Psychosom Med 2009;71:423-30.

28. Vetter ML, Wadden TA, Vinnard C, et al. Gender differences in the relationship between symptoms of depression and high-sensitivity CRP. Int J Obes (Lond) 2013;37(Suppl 1):S38-43.

29. Ford DE, Erlinger TP. Depression and C-reactive protein in US adults: data from the Third National Health and Nutrition Examination Survey. Arch Intern Med 2004;164:1010-14.

30. Macy EM, Hayes TE, Tracy RP. Variability in the measurement of C-reactive protein in healthy subjects: implications for reference intervals and epidemiological applications. Clin Chem 1997:43:52-8.

31. Pan A, Franco OH, Wang YF, et al. Prevalence and geographic disparity of depressive symptoms among middle-aged and elderly in China. J Affect Disord 2008;105:167-75.

32. Lu Y, Feng L, Feng L, et al. Systemic inflammation, depression and obstructive pulmonary function: a population-based study. Respir Res 2013;14:53. 